DOI: $10.12731 / 2070-7568-2021-10-2-132-145$

УДК 519.862.2, 51-77

\title{
ПОСТРОЕНИЕ И АНАЛИЗ \\ МАТЕМАТИЧЕСКОЙ МОДЕЛИ ЭКОНОМИЧЕСКИХ ПРОЦЕССОВ НА ОСНОВЕ СИСТЕМЫ ОДНОВРЕМЕННЫХ УРАВНЕНИЙ
}

\author{
Лажаунинкас Ю.В., Берднова Е.В.
}

В настоящее время народное хозяйство нашей страны нуждается в четкой и эффективной экономической модели с возможностью проведения анализа доли денежных средств на потребление и инвестиции, так как государство заинтересовано в скорейшем росте и развитии различных отраслей. Предлагаемая авторами статьи модель со статистически значимыми коэффициентами обладает высокой точностью, а значит и эффективностью.

Цель: используя конкретныле числовые данные, построить статическую модель Кейнса, определить параметры системы одновременных эконометрических уравнений, проанализировать получивиуюся модель.

Метод и методология: общенаучные методы теоретического исследования: анализ и синтез, систематизация, обобщение, методы регрессионного анализа.

Результаты: получена структурная форма экономической модели сельскохозяйственной отрасли Саратовской области. Построенная модель свидетельствует о том, что из каждой дополнительной тысячи дохода на потребление расходуется 777 ден. ед., а 223 ден. ед. инвестируется; дополнительные вложения в размере 1 тысячи ден. ед. приведут при прочих равных условиях к дополнительному увеличению потребления на 3,48 тыс. ден. ед.; дополнительные инвестиции в размере 1 тыс. ден. ед. приведут при прочих равных условиях к увеличению дополнительного дохода на 4,48 тыс. ден. ед. 
Область применения: результаты исследования могут быть использованы для оченки эффективности субсидирования различных отраслей народного хозяйства на уровне регионов, то есть для получения числовых значений денежных средств на потребление и инвестиции, а также для расчета инвестиционного мультипликатора потребления и инвестищионного мультипликатора начионального дохода.

Ключевые слова: экономическая модель; система одновременных уравнений; модель Кейнса; непрямой метод наименьших квадратов; регрессионный анализ; инвестищия; сельскохозяйственная отрасль

\section{CONSTRUCTION AND ANALYSIS OF A MATHEMATICAL MODEL OF ECONOMIC PROCESSES BASED ON SYSTEMS OF SIMULTANEOUS EQUATIONS}

\section{Lazhauninkas J.V., Berdnova E.V.}

Currently, the national economy of our country needs a clear and effective economic model with the ability to analyze the share of funds for consumption and investment, as the state is interested in the rapid growth and development of various industries. The model proposed by the authors with statistically significant coefficients has high accuracy, and therefore efficiency.

Purpose of the work is to construct a static Keynes model for describing the national economy of the country, to determine the parameters of the system of simultaneous econometric equations, and to analyze the resulting model.

The method or methodology of work: the article used general scientific methods of theoretical research: analysis and synthesis, systematization, generalization, methods of regression analysis.

Results: the structural form of the economic model of the agricultural sector of the Saratov region is obtained. The constructed model shows that out of each additional one thousand of income, 777 mon- 
etary units are spent on consumption, and 223 monetary units are invested; additional investments in the amount of 1 thousand monetary units will lead, all other things being equal, to an additional increase in consumption by 3,48 thousand monetary units; additional investments in the amount of 1 thousand monetary units will lead, all other things being equal, to an increase in additional income by 4,48 thousand monetary units.

Scope of results: the results of the study can be used to assess the effectiveness of subsidies to various sectors of the national economy at the regional level, that is, to obtain numerical values of funds for consumption and investment, as well as to calculate the investment multiplier of consumption and the investment multiplier of national income.

Keywords: economic model; system of simultaneous equations; Keynes model; indirect least squares method; regression analysis; investment; agricultural industry

\section{Введение}

Моделирование сложных экономических объектов зачастую предполагает ввод не одного, а нескольких уравнений, связанных между собой $[7,10,14,17]$. Таким образом, экономическая модель описывается системой уравнений. Подобные системы эконометрических уравнений довольно часто используются при построении моделей макроэкономики различных стран.

Построение экономической модели с применением системы одновременных уравнений имеет ряд сложностей $[5,7,10,12]$, которые часто связаны с ошибочностью спецификации модели. Особенностью построения является то, что аналитик может ошибочно выбрать модель для описания экономических процессов, в виду множества факторов, влияющих на экономические переменные. Теоретическое представление эндогенных и экзогенных переменных довольно часто не соответствует практическому состоянию моделируемого объекта. К тому же набор этих переменных может меняться, что, в свою очередь, влечет изменение вида модели с точки зрения идентифицируемости. 
Классическая модель экономики $[2,8,13,15]$ позволяет решить задачу поиска равновесия в условиях полной занятости. Но достаточно трудно достичь равновесия, когда экономика характеризуется безработицей.

Английский экономист Дж. М. Кейнс видел свою задачу в том, чтобы показать, что равновесие при полной занятости не является общим случаем. Общий случай - это равновесие при наличии безработицы, а полная занятость лишь особый случай. Чтобы достигнуть желаемого состояния полной занятости, государство обязано проводить особую политику по ее достижению, поскольку автоматически действующие рыночные силы без этой поддержки не гарантируют ее достижения [11]. Кейнс разработал модель макроэкономического равновесия, основанную на связи между совокупными доходами и расходами.

Поэтому большинство систем эконометрических уравнений, используемых для построения макроэкономических моделей различных стран, являются мультипликаторными моделями кейнсианского типа различной степени сложности $[3,5,9,10]$.

Рассматривая эти модели, следует отметить, что каждая в определенной мере отражает реальные связи и зависимости, но ни одна не претендует на общую теорию. Экономика любой страны, непрерывно развиваясь, становится все сложнее, поэтому любая модель со временем вступает в противоречие с многофакторной экономической реальностью.

Статическая модель Кейнса для описания народного хозяйства страны в наиболее простом варианте имеет следующий вид:

$$
\left\{\begin{array}{c}
C=a+b \cdot y+\varepsilon \\
y=C+I
\end{array}\right.
$$

где $C$ - личное потребление в постоянных ценах,

$y$ - национальный доход в постоянных ценах,

$I$ - инвестиции в постоянных ценах,

$\varepsilon-$ случайная составляющая.

Эта модель может быть использована и для описания различных отраслей, крупных корпораций, холдингов, групп компаний, 
ассоциаций, так как они оказывают большое влияние на социально-экономическое развитие отдельного региона и страны, в целом.

Актуальность данной статьи состоит в построении четкой и эффективной экономической модели с возможностью проведения анализа доли денежных средств на потребление и инвестиции.

Материалы и методы исследования. Используя усредненные данные по инвестированию сельскохозяйственной отрасли Саратовской области [18], указанные в таблице 1, построим систему одновременных уравнений. Определим параметры уравнений с помощью непрямого метода наименьших квадратов. Проанализируем получившуюся модель.

Таблииа 1.

Инвестирование сельскохозяйственной отрасли Саратовской области

\begin{tabular}{|c|c|c|}
\hline $\begin{array}{c}\text { Уровень производства и дохода, } \\
\text { млн. ден.ед. (у) }\end{array}$ & $\begin{array}{c}\text { Потребление, } \\
\text { млн. ден.ед. (С) }\end{array}$ & $\begin{array}{c}\text { Инвестиции, } \\
\text { млн. ден.ед. (I) }\end{array}$ \\
\hline 370 & 360 & 10 \\
\hline 415 & 395 & 20 \\
\hline 430 & 405 & 25 \\
\hline 455 & 428 & 27 \\
\hline 485 & 450 & 35 \\
\hline 490 & 454 & 36 \\
\hline 505 & 465 & 40 \\
\hline 520 & 478 & 42 \\
\hline 545 & 497 & 48 \\
\hline 570 & 514 & 56 \\
\hline
\end{tabular}

\section{Результаты и обсуждение}

По смыслу у и С - эндогенные переменные, I - экзогенная переменная. Любую систему одновременных уравнений можно представить в приведенной форме, коэффициенты которой оцениваются без затруднений. Но аналитики, как правило, используют коэффициенты структурной формы. В связи с этим возникает вопрос о возможности обратного перехода от приведенной формы к структурной. Именно в этом состоит сущность проблемы идентифицируемости систем эконометрических уравнений. 
Определим идентифицируемость первого уравнения. Уравнение идентифицируемо, если количество эндогенных переменных этого уравнения без единицы не больше разности общего количества экзогенных переменных системы и количества экзогенных переменных, входящих в первое уравнение.

Число эндогенных переменных, входящих в первое уравнение $n_{1}=$ 2 ; общее количество экзогенных переменных в системе $m=1$; количество $m_{1}=0$. Проверяем идентифицируемость с помощью правила: $n_{i}$ $1 \leq m-m_{i}$. Получаем 2-1=1-0, $1=1$. Тогда первое уравнение является точно идентифицируемым. Следовательно, и система одновременных уравнений точно идентифицируема, и можно использовать непрямой метод наименыших квадратов для определения параметров уравнений.

Приведенную форму модели получим путем преобразования уравнений структурной формы модели.

Из второго уравнения $y=C+I$. Подставим у в первое уравнение: $C=a+b \cdot(C+I)+\varepsilon$, тогда $C=\frac{a}{1-b}+\frac{b}{1-b} \cdot I+\frac{\varepsilon}{1-b}$.

Подставим значение $C$ из первого уравнения во второе: $y=\frac{a}{1-b}+\frac{1}{1-b} \cdot I+\frac{\varepsilon}{1-b}$.

Обозначим эндогенные переменные $C$ и $y$ через $Y 1$ и $Y 2$, а экзогенную переменную $I$ через $X 1$. Итак, систему одновременных уравнений в приведенной форме можно записать так:

$$
\begin{aligned}
& Y 1=b_{10}+b_{11} X 1+\varepsilon_{1}, \\
& Y 2=b_{20}+b_{21} X 1+\varepsilon_{2} .
\end{aligned}
$$

Здесь $b_{10}=b_{20}=\frac{a}{1-b}, b_{11}=\frac{b}{1-b}, b_{21}=\frac{1}{1-b}$.

$\mathrm{C}$ помощью метода наименьших квадратов найдем параметры каждого из уравнений этой системы.

Используя MS Excel, для первого уравнения получим следующее (рис. 1).

Проведем анализ полученных результатов.

Множественный $\mathrm{R}=0,994297$. Это означает, что между X1 и Y1 существует тесная линейная связь. Коэффициент детерминации $\mathrm{R}^{2}=0,987$. 


\begin{tabular}{|c|c|c|c|c|c|}
\hline вывод итогов & & & & & \\
\hline \multicolumn{6}{|c|}{ Регрессионная статистика } \\
\hline Множественный R & 0,994297489 & & & & \\
\hline R-квадрат & 0,988627497 & & & & \\
\hline Нормированный R-квадрат & 0,987205935 & & & & \\
\hline Стандартная ошибка & 5,420988417 & & & & \\
\hline Наблюдения & 10 & & & & \\
\hline \multicolumn{6}{|l|}{ Дисперсионный анализ } \\
\hline & $d f$ & SS & MS & $F$ & Значимость $F$ \\
\hline Регрессия & 1 & 20437,30308 & 20437,30308 & 695,4511454 & $4,59481 \mathrm{E}-09$ \\
\hline Остаток & 8 & 235,0969234 & 29,38711542 & & \\
\hline \multirow[t]{2}{*}{ Итого } & 9 & 20672,4 & & & \\
\hline & Коэффиициенты & $\begin{array}{c}\text { Стандартная } \\
\text { ошибка } \\
\end{array}$ & t-статистика & Р-значение & \\
\hline Y-пересечение & 326,6041259 & 4,791539131 & 68,16267529 & $2,38866 \mathrm{E}-12$ & \\
\hline $\mathrm{x} 1$ & 3,48070425 & 0,131987806 & 26,37140772 & $4,59481 \mathrm{E}-09$ & \\
\hline
\end{tabular}

Рис. 1. Расчет параметров первого уравнения

Для первого уравнения коэффициенты $b_{10}=326,6, b_{11}=3,48$. При проверке их статистической значимости по критерию Стьюдента, получаем следующие значения уровней значимости (Р-Значения): $\alpha_{b 10}=2,39 \cdot 10^{-12}, \alpha_{b 11}=4,59 \cdot 10^{-9}$. Так как эти значения меньше 0,05, то с вероятностью 0,95 можно утверждать, что оба коэффициента статистически значимы и могут быть включены в модель.

Таким образом, первое уравнение приведенной формы модели имеет вид: $\mathrm{Y} 1=326,6+3,48 \mathrm{X} 1+\varepsilon_{1}$. При проверке этого уравнения на адекватность по критерию Фишера получим $\alpha_{\text {Fнабл }}=4,59 \cdot 10^{-9}<0,05$. Тогда с вероятностью 0,95 можно утверждать, что уравнение адекватно исходным данным.

Произведем аналогичный расчет для второго уравнения приведенной формы модели (рис.2).

Множественный $\mathrm{R}=0,99654$. Это означает, что между X1 и $\mathrm{Y} 2$ существует тесная линейная связь. Коэффициент детерминации $\mathrm{R}^{2}=0,992$.

Коэффициенты для второго уравнения $b_{20}=326,6, b_{21}=4,48$. При проверке их статистической значимости по критерию Стьюдента, получаем следующие значения уровней значимости (Р-Значения): 
$a_{b 20}=2,39 \cdot 10^{-12}, a_{b 11}=6,19 \cdot 10^{-10}$. Так как эти значения меньше 0,05 , то с вероятностью 0,95 можно утверждать, что оба коэффициента статистически значимы и могут быть включены в модель.

\begin{tabular}{|c|c|c|c|c|c|}
\hline ВЫВОД ИТОГОВ & & & & & \\
\hline \multicolumn{6}{|c|}{ Регрессионная статистика } \\
\hline Множественный R & 0,996547123 & & & & \\
\hline R-квадрат & 0,993106167 & & & & \\
\hline Нормированный R-квадрат & 0,992244438 & & & & \\
\hline Стандартная ошибка & 5,420988417 & & & & \\
\hline Наблюдения & 10 & & & & \\
\hline \multicolumn{6}{|l|}{ Дисперсионный анализ } \\
\hline & $d f$ & SS & MS & $F$ & Значимость F \\
\hline Регрессия & 1 & 33867,40308 & 33867,40308 & 1152,457551 & $6,19301 \mathrm{E}-10$ \\
\hline Остаток & 8 & 235,0969234 & 29,38711542 & & \\
\hline \multirow[t]{2}{*}{ Итого } & 9 & 34102,5 & & & \\
\hline & Коэффициенты & $\begin{array}{c}\text { Стандартная } \\
\text { ошибка }\end{array}$ & t-статистика & Р-значение & \\
\hline Ү-пересечение & 326,6041259 & 4,791539131 & 68,16267529 & $2,38866 \mathrm{E}-12$ & \\
\hline $\mathrm{x} 1$ & 4,48070425 & 0,131987806 & 33,9478652 & $6,19301 \mathrm{E}-10$ & \\
\hline
\end{tabular}

Рис. 2. Расчет параметров второго уравнения

Получаем второе уравнение приведенной формы модели: $\mathrm{Y} 2=326,6+4,48 \mathrm{X} 1+\varepsilon_{2}$. При проверке этого уравнения на адекватность по критерию Фишера получим $\alpha_{F \text { наб }}=6,19 \cdot 10^{-10}<0,05$. Так как, то с вероятностью 0,95 можно утверждать, что уравнение адекватно исходным данным.

Итак, приведенная форма модели:

$$
\begin{aligned}
& \mathrm{Y} 1=326,6+3,48 \times 1+\varepsilon_{1}, \\
& \mathrm{Y} 2=326,6+4,48 \times 1+\varepsilon_{2}
\end{aligned}
$$

Перейдем от приведенной формы модели к структурной. Как было отмечено ранее, $b_{10}=b_{20}=\frac{a}{1-b}, b_{11}=\frac{b}{1-b}, b_{21}=\frac{1}{1-b}$.

Подставим полученные значения $b_{10}, b_{20}, b_{11}, b_{21}$ : $\frac{a}{1-b}=326,6, \frac{b}{1-b}=3,48, \frac{1}{1-b}=4,48$.

Тогда $a=72,83, b=0,777$.

Запишем структурную форму модели: 


$$
\left\{\begin{array}{c}
C=72,83+0,777 \cdot y+\varepsilon, \\
y=C+I .
\end{array}\right.
$$

Структурный коэффициент b характеризует предельную склонность к потреблению - это коэффициент, который показывает, на сколько изменится потребление при росте или сокращении дохода на единицу. $b=0,777$ - означает, что из каждой дополнительной тысячи дохода на потребление расходуется 777 ден. ед., а 223 ден. ед. инвестируется. Предельная склонность к потреблению находится в интервале от 0 до 1. Это свидетельствует о стабильности роста дохода и уровня потребления.

Склонность к потреблению может оказаться больше единицы, если происходит временное снижение дохода и берется займ для того, чтобы сохранить уровень потребления.

Склонность к потреблению влияет на величину мультипликатора. Чем выше склонность к потреблению, тем большая часть дополнительного дохода расходуется и тем сильнее будет мультипликативный эффект. В данном случае коэффициент $b_{11}=\frac{b}{1-b}=3,48$ представляет собой инвестиционный мультипликатор потребления. Эта величина показывает, что дополнительные вложения в размере 1 тысячи денежных единиц приведут при прочих равных условиях к дополнительному увеличению потребления на 3,48 тыс. ден. ед.

Коэффициент $b_{21}=\frac{1}{1-b}=4,48-$ инвестиционный мультипликатор регионального дохода. Он показывает, что дополнительные инвестиции в размере 1 тыс. ден. ед. приведут при прочих равных условиях к увеличению дополнительного дохода на 4,48 тыс. ден. ед.

Преимуществом предлагаемой модели является отказ от набора относительных показателей, отражающих динамику производства отдельных видов продукции на единицу полученных субсидий.

\section{Заключение}

Итак, мы получили модель со статистически значимыми коэффициентами. Проверка уравнений на адекватность по критерию 
Фишера дала достаточно высокий результат, что говорит о высокой точности построенной модели.

Практическая значимость проведенного расчета заключается в получении структурной формы экономической модели сельскохозяйственной отрасли Саратовской области с возможностью проведения анализа доли денежных средств на потребление и инвестиции, а также для расчета инвестиционного мультипликатора потребления и инвестиционного мультипликатора регионального дохода.

Авторы не претендуют на уникальность предлагаемой модели. Предложенный алгоритм построения модели и расчета ее параметров является универсальным и может быть использован для оценки эффективности субсидирования различных отраслей народного хозяйства на уровне регионов.

\section{Список литературы}

1. Берднова Е.В., Лажаунинкас Ю.В. Цифровое моделирование производства пшеницы и сои на основе экономико-математических методов // Наука Красноярья. 2019. Т. 8. № 4. С. 7-19.

2. Гончаров В.Д., Сальников С.Г. Моделирование экономических процессов в агропромышленном комплексе // Инвестиции в России. 2019. № 8 (295). С. 9-12.

3. Кинякин В.Н., Слесарева Е.А. Системы регрессионных (одновременных) уравнений // Вестник экономической безопасности. 2016. № 4. С. 265-270.

4. Киселев Н.И., Алексахина В.Г. Оценка качества эконометрических моделей в виде системы одновременных уравнений // Вопросы региональной экономики. 2014. № 1 (18). С. 49-56.

5. Колодняя Г.В. Возможность применения кейнсианских идей при решении проблем современной российской экономики // Теоретическая экономика. 2019. № 1 (49). С. 10-15.

6. Кузнецова Э.Р., Антинескул Е.А. Моделирование процесса формирования местного бюджета и показателей социально-экономического развития в среднесрочной перспективе // Экономика и предпринимательство. 2020. № 9 (122). С. 596-599. 
7. Лисова Е.В. Моделирование социально-экономических процессов региона // Путеводитель предпринимателя. 2020. Т. 13. № 1. С. 130-136.

8. Мельникова Ю.В., Лажаунинкас Ю.В. Математическое моделирование экономической конъюнктуры российского зернового рынка на основе трехпараметрической модели Хольта-Винтерса // Вестник Алтайской академии экономики и права. 2020. № 11-2. С. 280-288.

9. Попова С.В. Применение кейнсианской теории к решению проблем современного экономического развития России // Финансовая экономика. 2019. № 11. С. 57-60.

10. Селезнев И.А. Моделирование экономических процессов как методы анализа сложных систем // Международная конференция по мягким вычислениям и измерениям. 2017. Т. 3. С. 131-134.

11. Харрис Л. Денежная теория. М.: Прогресс, 1990. С.269

12. Царегородцев Е.И. Проблемы моделирования экономических процессов // Актуальные проблемы экономики современной России. 2017. № 4. C. 119-122.

13. Шаяхметов Д.Ф. Сравнительный анализ подходов к моделированию экономических процессов // Наука Красноярья. 2017. Т. 6. № 4-2. С. 60-69.

14. Mezhevich N. The Baltic economic model: some results of the 19902015 transformations // Baltic Region. 2015. No 4(26). Pp. 20-37. DOI 10.5922/2079-8555-2015-4-2.

15. Gulaliyev M., Abasova S., Mehdiyeva I., Sultanova N. Model shaping forms of state intervention in the economy and economic model of the country // International Journal of Management and Business Research. 2018. Vol. 8. No 1. Pp. 80-92.

16. Sevastyanova E.P., Giese R. Replacement of dominating paradigm as outcome of the current economic crisis // Journal of Siberian Federal University. Humanities and Social Sciences. 2011. Vol. 4. No 5. Pp. 722-733.

17. Tkachev S.I., Berdnova E.V., Rubtsova S.N., Pakhomova T.V., Lazhauninkas Yu.V., Sleptsova L.A. Economic mathematical modeling of agrarian industry development by cluster analysis // Revista Turismo Estudos \& Práticas. 2020. № S1. Pp. 53-55.

18. Субсидии на развитие сельского хозяйства [Электронный ресурс] // Министерство сельского хозяйства Саратовской области. URL: https:// www.minagro.saratov.gov.ru/subsidii/ (дата обращения 10.05.2021). 


\section{References}

1. Berdnova E.V., Lazhauninkas Yu.V. Tsifrovoe modelirovanie proizvodstva pshenitsy i soi na osnove ekonomiko-matematicheskikh metodov [Digital modeling of wheat and soybean production based on economic and mathematical methods]. Nauka Krasnoyar'ya, 2019, vol. 8, no. 4, pp. 7-19.

2. Goncharov V.D., Sal'nikov S.G. Modelirovanie ekonomicheskikh protsessov v agropromyshlennom komplekse [Modeling of economic processes in the agro-industrial complex]. Investitsii v Rossii, 2019, no. 8 (295), pp. 9-12.

3. Kinyakin V.N., Slesareva E.A. Sistemy regressionnykh (odnovremenny$\mathrm{kh}$ ) uravneniy [Systems of regression (simultaneous) equations]. Vestnik ekonomicheskoy bezopasnosti, 2016, no. 4, pp. 265-270.

4. Kiselev N.I., Aleksakhina V.G. Otsenka kachestva ekonometricheskikh modeley $\mathrm{v}$ vide sistemy odnovremennykh uravneniy [Evaluation of the quality of econometric models in the form of a system of simultaneous equations]. Voprosy regional'noy ekonomiki, 2014, no. 1 (18), pp. 49-56.

5. Kolodnyaya G.V. Vozmozhnost' primeneniya keynsianskikh idey pri reshenii problem sovremennoy rossiyskoy ekonomiki [The possibility of applying Keynesian ideas in solving the problems of the modern Russian economy]. Teoreticheskaya ekonomika, 2019, no. 1 (49), pp. 10-15.

6. Kuznetsova E.R., Antineskul E.A. Modelirovanie protsessa formirovaniya mestnogo byudzheta i pokazateley sotsial'no-ekonomicheskogo razvitiya $\mathrm{v}$ srednesrochnoy perspektive [Modeling the process of forming the local budget and indicators of socio-economic development in the medium term]. Ekonomika i predprinimatel'stvo, 2020, no. 9 (122), pp. 596-599.

7. Lisova E.V. Modelirovanie sotsial'no-ekonomicheskikh protsessov regiona [Modeling of socio-economic processes in the region]. Putevoditel'predprinimatelya, 2020, vol. 13, no. 1, pp. 130-136.

8. Mel'nikova Yu.V., Lazhauninkas Yu.V. Matematicheskoe modelirovanie ekonomicheskoy kon"yunktury rossiyskogo zernovogo rynka na osnove trekhparametricheskoy modeli Khol'ta-Vintersa [Mathematical modeling of the economic situation of the Russian grain market based on the Holt-Winters three-parameter model]. Vestnik Altayskoy akademii ekonomiki i prava, 2020, no. 11-2, pp. 280-288. 
9. Popova S.V. Primenenie keynsianskoy teorii k resheniyu problem sovremennogo ekonomicheskogo razvitiya Rossii [Application of Keynesian theory to solving the problems of modern economic development in Russia]. Finansovaya ekonomika, 2019, no. 11, pp. 57-60.

10. Seleznev I.A. Modelirovanie ekonomicheskikh protsessov kak metody analiza slozhnykh sistem [Modeling of economic processes as methods of analysis of complex systems]. Mezhdunarodnaya konferentsiya po myagkim vychisleniyam i izmereniyam [International Conference on Soft Computing and Measurements]. 2017, vol. 3, pp. 131-134.

11. Kharris L. Denezhnaya teoriya [Monetary Theory]. Moscow: Progress, 1990, pp. 269.

12. Tsaregorodtsev E.I. Problemy modelirovaniya ekonomicheskikh protsessov [Problems of modeling economic processes]. Aktual'nye problemy ekonomiki sovremennoy Rossii, 2017, no. 4, pp. 119-122.

13. Shayakhmetov D.F. Sravnitel'nyy analiz podkhodov k modelirovaniyu ekonomicheskikh protsessov [Comparative analysis of approaches to modeling economic processes]. Nauka Krasnoyar 'ya, 2017, vol. 6, no. 4-2, pp. 60-69.

14. Mezhevich, N. The Baltic economic model: some results of the 19902015 transformations. Baltic Region, 2015, no. 4(26), pp. 20-37. DOI: https://doi.org/10.5922/2079-8555-2015-4-2

15. Gulaliyev M., Abasova S., Mehdiyeva I., Sultanova N. Model shaping forms of state intervention in the economy and economic model of the country. International Journal of Management and Business Research, 2018, vol. 8, no. 1, pp. 80-92.

16. Sevastyanova E.P., Giese R. Replacement of dominating paradigm as outcome of the current economic crisis. Journal of Siberian Federal University. Humanities and Social Sciences, 2011, vol. 4, no. 5, pp. $722-733$.

17. Tkachev S.I., Berdnova E.V., Rubtsova S.N., Pakhomova T.V., Lazhauninkas Yu.V., Sleptsova L.A. Economic mathematical modeling of agrarian industry development by cluster analysis. Revista Turismo Estudos \& Práticas, 2020, no. S1, pp. 53-55.

18. Subsidii na razvitie sel'skogo khozyaystva [Subsidies for agricultural development]. Ministry of Agriculture of the Saratov region] https:// 
www.minagro.saratov.gov.ru/subsidii/poluchateli-subsidiy (accessed 10.05.2021).

\section{ДАННЫЕ ОБ АВТОРАХ}

Лажаунинкас Юлия Владимировна, доцент кафедры «Экономическая кибернетика», кандидат педагогических наук ФГБОУ ВО Саратовский государственный аграрньй университет имени Н.И. Вавилова

Театральная пл., 1, г. Саратов, 410012, Российская Федераџия lazhauninkas@yandex.ru

Берднова Екатерина Владимировна, доцент кафедры «Экономическая кибернетика», кандидат педагогических наук ФГБОУ ВО Саратовский государственный аграрный университет имени Н.И. Вавилова

Театральная пл., 1, г. Саратов, 410012, Российская Федерация berdnovsn@mail.ru

DATA ABOUT THE AUTHORS

Lazhauninkas Julia V., Associate Professor «Economic Cybernetics», Candidate of Pedagogical Sciences Saratov State Agrarian University

1, Teatral'naya Sq., Saratov, 410012, Russian Federation lazhauninkas@yandex.ru

SPIN-code: 8735-6760

ORCID: 0000-0002-8018-7818

Berdnova Ekaterina V., Associate Professor «Economic Cybernetics», Candidate of Pedagogical Sciences Saratov State Agrarian University

1, Teatral'naya Sq., Saratov, 410012, Russian Federation berdnovsn@mail.ru

SPIN-code: 4366-2619

ORCID: 0000-0002-2448-1545 